\title{
Histopathologic findings in breast surgical specimens from patients undergoing female-to-male gender reassignment surgery
}

\author{
Vanda F. Torous ${ }^{1,2} \cdot$ Stuart J. Schnitt ${ }^{2,3,4}$ \\ Received: 22 April 2018 / Revised: 21 June 2018 / Accepted: 3 August 2018 / Published online: 11 October 2018 \\ (c) United States \& Canadian Academy of Pathology 2018
}

\begin{abstract}
Breast reduction surgery or mastectomy following administration of androgen therapy is part of the female-to-male gender reassignment process. Details regarding the histopathologic findings in breast tissue from patients undergoing female-tomale gender reassignment surgery are limited. We reviewed hematoxylin and eosin-stained sections of breast tissue from 148 patients who underwent breast reduction surgery or mastectomy as part of the female-to-male gender reassignment process at our institution between January 2014 and May 2017. The spectrum of histologic features in each case was catalogued. The median patient age was 27 years (range 18-60 years). Lobular atrophy was seen to some degree in $73 \%$ of cases and was prominent in $42 \%$. A predominantly fibrotic stroma was seen in $45 \%$ of cases and areas resembling the fibrous stage of gynecomastia were seen in $41 \%$. Other features included variably ectatic ducts in $96 \%$ of cases, cysts in $42 \%$, apocrine metaplasia in $32 \%$, fibroadenomatous change in $27 \%$, usual ductal hyperplasia in $26 \%$, and pseudoangiomatous stromal hyperplasia in $19 \%$. Five cases $(3 \%)$ demonstrated atypical hyperplasia (atypical ductal hyperplasia in 2 ; atypical lobular hyperplasia in 2; both atypical ductal hyperplasia and atypical lobular hyperplasia in 1). One case demonstrated high grade ductal carcinoma in situ. No invasive carcinomas were identified. In conclusion, the majority of breast specimens from patients undergoing female-to-male gender reassignment demonstrate at least some degree of lobular atrophy as well as ectatic ducts, fibrous stroma, and areas resembling the fibrous stage of gynecomastia. Only rare cases showed atypical lesions; the clinical significance of these lesions in this setting is uncertain.
\end{abstract}

\section{Introduction}

At the present time an estimated $0.4-0.6 \%$ of adults, or about 1-1.4 million people in the United States, identify as transgender [1, 2]. In 2016, there were more than 3250 gender reassignment surgeries performed in the United States alone, about a 19\% increase since the previous year [3]. About half of gender reassignment surgeries were female-to-male [3]. Female-to-male gender reassignment

Stuart J. Schnitt

sschnitt@bwh.harvard.edu

1 Department of Pathology, Massachusetts General Hospital, Boston, MA, USA

2 Department of Pathology, Harvard Medical School, Boston, MA, USA

3 Department of Pathology, Brigham and Women's Hospital, Boston, MA, USA

4 Dana-Farber Cancer Institute, Boston, MA, USA surgery includes a number of complex surgical procedures, including facial masculinization surgery, phalloplasty, and mastectomy. Surgical removal of the breast tissue is both the first and the most common surgical procedure requested in the female-to-male transition process $[4,5]$. In addition to breast surgery, androgen administration is typically employed for the masculinization process.

To date, few studies have detailed the histopathologic findings in breast specimens of patients undergoing femaleto-male gender reassignment. Grynberg et al. evaluated 100 bilateral mastectomy specimens from transgender patients and demonstrated a marked reduction of glandular tissue and lobular atrophy [6]; however, a more detailed pathologic description was not provided. Several additional studies have assessed the effects of androgens on the breast tissue of transgender patients, but were limited by small sample size making it difficult to draw firm conclusions [7-10]. One recent study characterized the clinical and immunophenotypic findings in 68 cases of female-tomale breast resection specimens [11]. In that study, fibrocystic changes were the most common histologic findings, 
followed by fibrotic stage gynecomastoid change; only one case in the series showed an atypical histologic alteration (flat epithelial atypia) [11].

To document further the spectrum of pathologic changes in breast tissue from this unique patient population, we reviewed breast surgical specimens from 148 patients who underwent mastectomy or breast reduction surgery at our institution as part of the female-to-male gender reassignment process.

\section{Materials and methods}

We searched the archives of the Department of Pathology at Beth Israel Deaconess Medical Center (Boston, MA) for breast specimens from patients with a clinical history of gender dysphoria. A total of 148 cases were identified between January 2014 and May 2017, with the number of cases increasing steadily over the study time period (Fig. 1). Specimen types included bilateral reduction mammoplasties $(n=138)$ and bilateral mastectomies $(n=$ 10). For comparison we also evaluated 50 consecutive bilateral reduction mammoplasty specimens from women under the age of 40 years accessioned during the same time period.

All hematoxylin and eosin-stained slides for each case were reviewed for a variety of histologic alterations (Table 1). Each case was assessed for the extent of lobular atrophy, defined as a decrease in the number and size of the acini per lobule, typically accompanied by replacement of

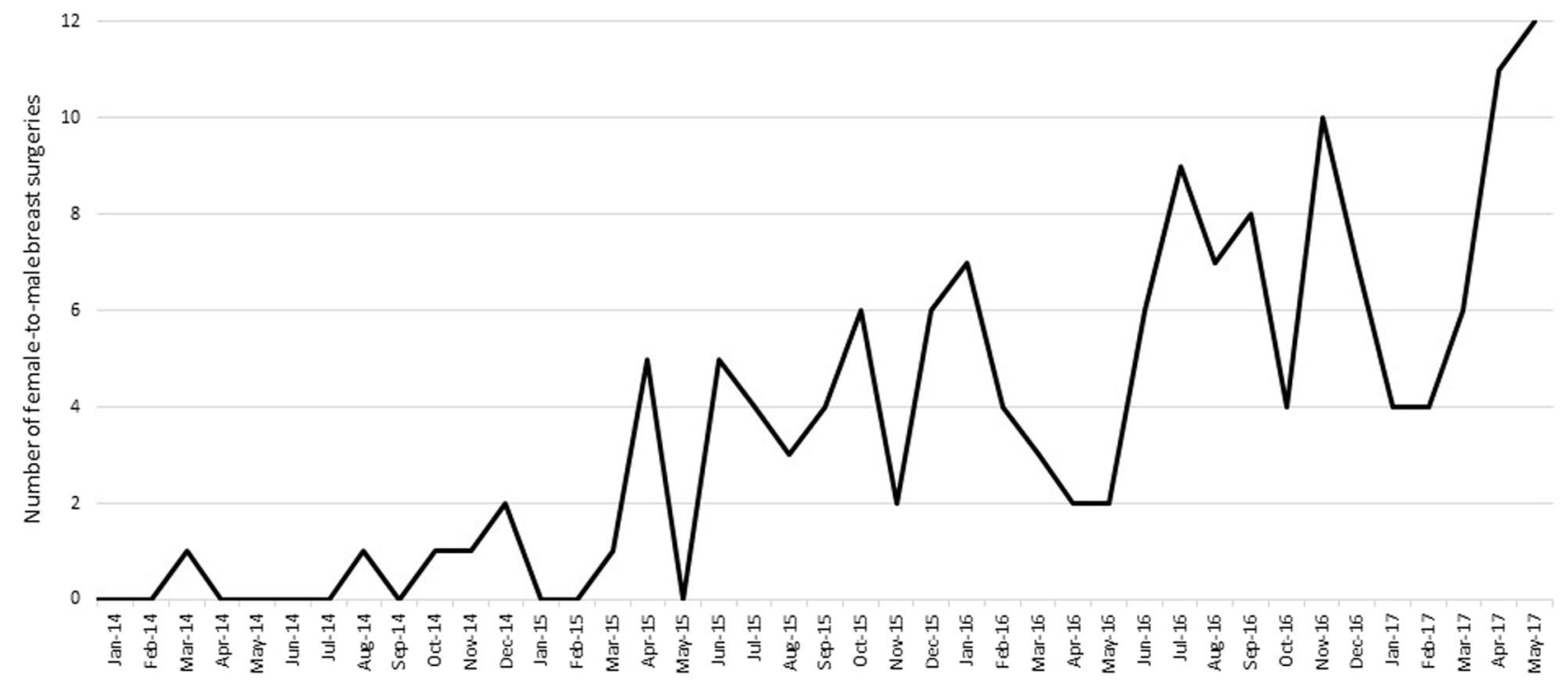

Fig. 1 Number of breast reduction operations (reduction mammoplasties, mastectomies) in female-to-male transsexual patients at Beth Israel Deaconess Medical Center, Boston, MA between January 2014 and May 2017

Table 1 Histologic features evaluated

\begin{tabular}{lll}
\hline Lobular atrophy & Benign lesions & Atypical lesions \\
Minimal (0-25\%) & Cysts & Flat epithelial atypia \\
Mild (26-50\%) & Apocrine metaplasia/cysts & Atypical ductal hyperplasia \\
Moderate (51-75\%) & Fibroadenomatous change & Atypical lobular hyperplasia \\
Marked (76-100\%) & Fibroadenoma & In-situ carcinoma (LCIS, DCIS) \\
Stroma & Papillomas & Invasive carcinoma (IDC, ILC) \\
Fibrous & Sclerosing adenosis & \\
Fatty & Gynecomastoid change & \\
Mixed & Columnar cell change & \\
Ectatic Ducts & Usual ductal hyperplasia & \\
Absent & Secretory change & \\
Scattered & Pseudoangiomatous stromal hyperplasia & \\
Abundant & Benign vascular lesions & \\
& Inflammation (mild and above) & \\
& Calcifications & \\
\hline
\end{tabular}


the specialized intralobular stroma with dense collagen [12]. Lobular atrophy was also characterized by an overall reduction in the number of terminal duct lobular units and was graded as minimal (0-25\% lobular involution), mild (26-50\%), moderate $(51-75 \%)$, and marked $(>75 \%)$. The stromal composition was also evaluated and categorized as predominantly fibrous, predominantly fatty, or mixed (when there were equal proportions of fibrous and fatty stroma). Stromal inflammation was categorized as none/ minimal, mild, moderate, and severe.

Each case was additionally assessed for the presence or absence of a spectrum of benign to malignant histologic alterations. Benign alterations included usual ductal hyperplasia, papillomas, sclerosing adenosis, fibroadenomas, fibroadenomatous change, gynecomastoid change, columnar cell change, secretory change, apocrine metaplasia/apocrine cysts, non-apocrine cysts, ectatic ducts, pseudoangiomatous stromal hyperplasia (PASH), benign vascular lesions, and calcifications. The extent of ectatic ducts was graded semi-quantitatively as absent, scattered, and abundant. The presence of atypical lesions (flat epithelial atypia, atypical ductal hyperplasia, atypical lobular hyperplasia), in situ carcinomas (ductal carcinoma in situ, lobular carcinoma in situ), and invasive cancers was also noted. As per our department protocol, sampling of reduction mammoplasty specimens consisted of two tissue blocks from each breast (i.e., four blocks/case), with additional sections submitted if a gross lesion or histologic atypia were identified. No additional sections or ancillary testing was performed as a part of this study.

The frequency of histologic features in the gender dysphoria group and the comparison group were compared using chi square and Fisher exact tests, as appropriate. Two-sided $p$-values of $<0.05$ were considered statistically significant. The study was approved by the Beth Israel Deaconess Medical Center Committee for Clinical Investigations.

\section{Results}

Among patients in the gender dysphoria group, the median and mean patient ages were 27 and 28.4 years, respectively, with an age range of 18-60 years. The median specimen weight (right and left breast combined) was $942 \mathrm{~g}$ (range 70-3252) and the median total number of tissue blocks submitted per case was 4 (range 4-69). Eighty-eight percent of patients underwent androgen therapy prior to gender reassignment surgery. Duration of androgen therapy ranged from 3 months to over 5 years prior to breast surgery.

The histologic findings are summarized in Table 2. The predominant histologic alteration was ectatic ducts, with almost all cases $(96 \%)$ demonstrating at least some degree and $49 \%$ demonstrating abundant ectatic ducts. At least mild lobular atrophy was seen in $73 \%$ of cases with $12 \%$ demonstrating marked lobular atrophy (Fig. 2). The stroma was predominantly fibrous in $45 \%$ of cases, predominantly fatty in 20\%, and mixed in 35\% (Fig. 3). Areas resembling the fibrous (or inactive) phase of gynecomastia were present in $41 \%$ of the cases (Fig. 4). Other histologic findings included non-apocrine cysts $(42 \%)$, apocrine metaplasia or apocrine cysts (32\%), fibroadenomatous change (27\%), usual ductal hyperplasia (26\%), PASH (19\%), and benign vascular lesions (benign hemangiomas and perilobular hemangiomas; 8\%) (Fig. 4). Calcifications were seen in $22 \%$ of cases, predominantly associated with lobules.

The breast specimens from 5 patients (3\%) demonstrated atypical lesions: 2 with atypical ductal hyperplasia, 2 with atypical lobular hyperplasia, and 1 with both atypical ductal hyperplasia and atypical lobular hyperplasia (Fig. 5). Additionally, 1 case demonstrated ductal carcinoma in situ (Fig. 6). The ductal carcinoma in situ in this case was high nuclear grade with comedo necrosis and was present in 2 of 45 blocks examined. Of note the ductal carcinoma in situ lesion demonstrated focal squamous differentiation.

The histologic findings in reduction mammoplasty cases from the age-matched comparison group are summarized in Table 2. Lobular atrophy, predominantly fibrous stroma, abundant ectatic ducts, gynecomastoid change, fibroadenomatous change and calcifications were all significantly less frequent in this group than among the gender dysphoria cases.

\section{Discussion}

The number of patients with gender dysphoria seeking gender reassignment surgery is increasing. As a result, surgical pathologists will be encountering breast specimens from these patients with increasing frequency. Several prior studies have described the complex surgical techniques required for the gender reassignment process, but studies assessing in detail the pathologic findings in the tissues of these patients, particularly breast tissue, have been few in number. To our knowledge, this is the largest study to date providing a detailed review of histologic alterations in breast tissue from this patient population.

One of the major histologic findings seen in our cases was lobular atrophy. The majority of cases (73\%) demonstrated more than just minimal involution of the lobules (defined as $>25 \%$ of lobules demonstrating atrophic changes). Additionally, the stroma in these cases was predominantly fibrous in quality. Prior studies that examined the breast tissues from female-to-male transsexual patients demonstrated similar findings with regard to lobular atrophy and stromal fibrosis. Sapino et al described the histologic 
Table 2 Clinical and histologic features of breast specimens from patients with gender dysphoria and age-matched comparison group of reduction mammoplasty specimens

\begin{tabular}{|c|c|c|c|}
\hline & Gender dysphoria cases $n=148$ & $\begin{array}{l}\text { Age-matched comparison } \\
\text { group } n=50\end{array}$ & $p$ value \\
\hline \multicolumn{4}{|l|}{ Clinical features } \\
\hline Age, median & 27.0 years (range $18-60$ ) & 27.0 years (range $18-38$ ) & \\
\hline Specimen weight, grams: mean; median & 1088; 942 (range 70-3252) & $1184 ; 1120$ (range $60-2974)$ & \\
\hline Cassettes evaluated per case: mean; median & $6.1 ; 4.0$ (range $4-69)$ & 4.0 (All cases) & \\
\hline Androgen therapy & $88 \%(124 / 148)$ & None & \\
\hline \multicolumn{4}{|l|}{ Histologic features } \\
\hline Lobular atrophy & & & $<0.0001$ \\
\hline Minimal $(0-25 \%)$ & $27 \%(40 / 148)$ & $96 \%(48 / 50)$ & \\
\hline Mild (26-50\%) & $31 \%(46 / 148)$ & $2 \%(1 / 50)$ & \\
\hline Moderate $(51-75 \%)$ & $30 \%(45 / 148)$ & $2 \%(1 / 50)$ & \\
\hline Marked (76-100\%) & $12 \%(17 / 148)$ & $0 \%(0 / 50)$ & \\
\hline \multicolumn{4}{|l|}{ Stroma } \\
\hline Fibrous & $45 \%(67 / 148)$ & $10 \%(5 / 50)$ & $<0.001$ \\
\hline Fatty & $20 \%(29 / 148)$ & $60 \%(30 / 50)$ & \\
\hline Mixed & $35 \%(52 / 148)$ & $30 \%(15 / 50)$ & \\
\hline Ectatic ducts & & & $<0.00001$ \\
\hline Absent & $4 \%(6 / 148)$ & $32 \%(16 / 50)$ & \\
\hline Scattered & $47 \%(70 / 148)$ & $34 \%(17 / 50)$ & \\
\hline Abundant & $49 \%(72 / 148)$ & $34 \%(17 / 50)$ & \\
\hline \multicolumn{4}{|l|}{ Atypical lesions } \\
\hline Flat epithelial atypia & $0 \%(0 / 148)$ & $0 \%(0 / 50)$ & NS \\
\hline Atypical ductal hyperplasia & $2 \%(3 / 148)$ & $0 \%(0 / 50)$ & NS \\
\hline Atypical lobular hyperplasia & $2 \%(3 / 148)$ & $0 \%(0 / 50)$ & NS \\
\hline In-situ carcinoma (LCIS, DCIS) & $1 \%(1 / 148)$ & $0 \%(0 / 50)$ & NS \\
\hline Invasive carcinoma (IDC, ILC) & $0 \%(0 / 148)$ & $0 \%(0 / 50)$ & NS \\
\hline \multicolumn{4}{|l|}{ Benign lesions } \\
\hline Gynecomastoid change & $41 \%(60 / 148)$ & $0 \%(0 / 50)$ & $<0.0001$ \\
\hline Cysts & $42 \%(62 / 148)$ & $20 \%(10 / 50)$ & 0.047 \\
\hline Apocrine metaplasia/apocrine cysts & $32 \%(47 / 148)$ & $34 \%(17 / 50)$ & NS \\
\hline Fibroadenomatous change & $27 \%(40 / 148)$ & $8 \%(4 / 50)$ & 0.02 \\
\hline Fibroadenoma & $3 \%(4 / 148)$ & $2 \%(1 / 50)$ & NS \\
\hline Usual ductal hyperplasia & $26 \%(38 / 148)$ & $12 \%(6 / 50)$ & NS \\
\hline Pseudoangiomatous stromal hyperplasia & $19 \%(28 / 148)$ & $18 \%(9 / 50)$ & NS \\
\hline Benign vascular lesions & $8 \%(12 / 148)$ & $0 \%(0 / 50)$ & NS \\
\hline Secretory change & $4 \%(6 / 148)$ & $2 \%(1 / 50)$ & NS \\
\hline Columnar cell change & $3 \%(5 / 148)$ & $2 \%(1 / 50)$ & NS \\
\hline Papillomas & $2 \%(3 / 148)$ & $0 \%(0 / 50)$ & NS \\
\hline Sclerosing adenosis & $1 \%(1 / 148)$ & $0 \%(0 / 50)$ & NS \\
\hline Calcifications & $22 \%(32 / 148)$ & $6 \%(3 / 50)$ & 0.03 \\
\hline Inflammation (mild and above) & $24 \%(36 / 148)$ & $14 \%(7 / 50)$ & NS \\
\hline
\end{tabular}

NS not significant

findings in two cases of transsexual patients who had undergone long-term androgen administration, and both demonstrated stromal sclerosis and epithelial atrophy [9]. Slagter et al. in a study of 23 female-to-male transsexual patients, documented both a marked reduction of glandular tissue as well as a prominence of fibrous connective tissue in their cases [7]. In their study of 100 breast specimens from transsexual patients, Grynberg et al reported that $93 \%$ of their cases showed marked reduction of glandular tissue and a proliferation of fibrous connective tissue [6]. In 
contrast, Burgess and Shousha examined the breast tissue of 29 female-to-male transsexual patients who had undergone long-term androgen therapy and did not find any significant differences in the prevalence of normal acini, ducts, and fibrosis between specimens from transsexual patients and those from normal controls [8].

In this study, we also documented other benign histologic alterations within the breast tissue of patients with gender dysphoria. Additional microscopic findings included ectatic ducts, cysts, fibroadenomatous change, apocrine metaplasia, PASH, and benign vascular lesions. Calcifications were also found frequently within the breast tissue of the gender dysphoric patients, which has been documented before [8]. Notably, many of our cases (41\%) demonstrated changes similar to those seen in the fibrous or inactive phase of gynecomastia. When compared with an age-matched group of women undergoing reduction mammoplasties, cases in the gender dysphoria group showed a significantly greater frequency of lobular atrophy, and a significantly greater proportion of cases showed gynecomastoid change, a predominantly fibrous stroma, abundant ectatic ducts, fibroadenomatous change and

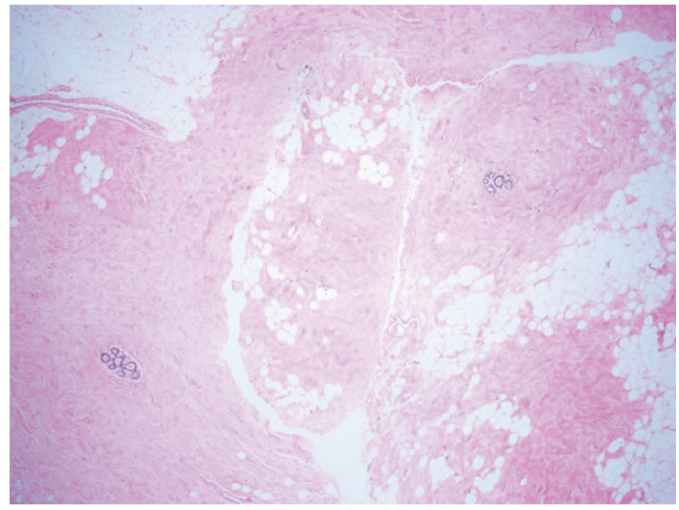

Fig. 2 Low power view of breast tissue from a patient with gender dysphoria demonstrating marked $(>75 \%)$ lobular atrophy calcifications. No cases in this comparison group demonstrated atypical lesions. The study by East and colleagues also demonstrated an increased prevalence of the fibrotic stage of gynecomastoid change in resection specimens from gender dysphoric patients with $32.4 \%$ of their patient cohort showing this histologic alteration [11]. These changes likely reflect the complex interaction of androgens and estrogens, both exogenously and endogenously derived, on the breast tissues of these patients.

In contrast to most prior studies, we observed not only benign epithelial proliferative alterations, but also atypical lesions including atypical ductal hyperplasia, atypical lobular hyperplasia, and ductal carcinoma in situ. Epidemiological and clinical studies have shown mixed results regarding the relationship between androgen administration and the risk of developing breast cancer. In our study, we found one case of ductal carcinoma in situ. That patient was 29 years old, had a family history of breast carcinoma in the patient's father, and histologically the ductal carcinoma in situ was high grade and demonstrated comedo necrosis and focal squamous differentiation. No imaging was performed prior to breast surgery, which is in line with normal protocol at our institution. Thus, although only a minority of our patients demonstrated atypical lesions (3\%) and in-situ carcinoma (1\%) with no invasive carcinomas identified, continued examination of the tissue from these patients is needed as the interactions of androgens with breast tissue in the complex hormonal milieu of these patients is still poorly understood. Additionally, it may be prudent in gender dysphoric patients with a family history of breast cancer to perform imaging studies prior to surgery as well as to examine additional sections of tissue.

The interaction of androgen receptors, estrogen receptors, and progesterone receptors and their effect on breast tissue has been unclear, with some studies demonstrating increased risk of developing breast carcinoma while others not finding such an association [13-24]. Additionally, while the assumption is
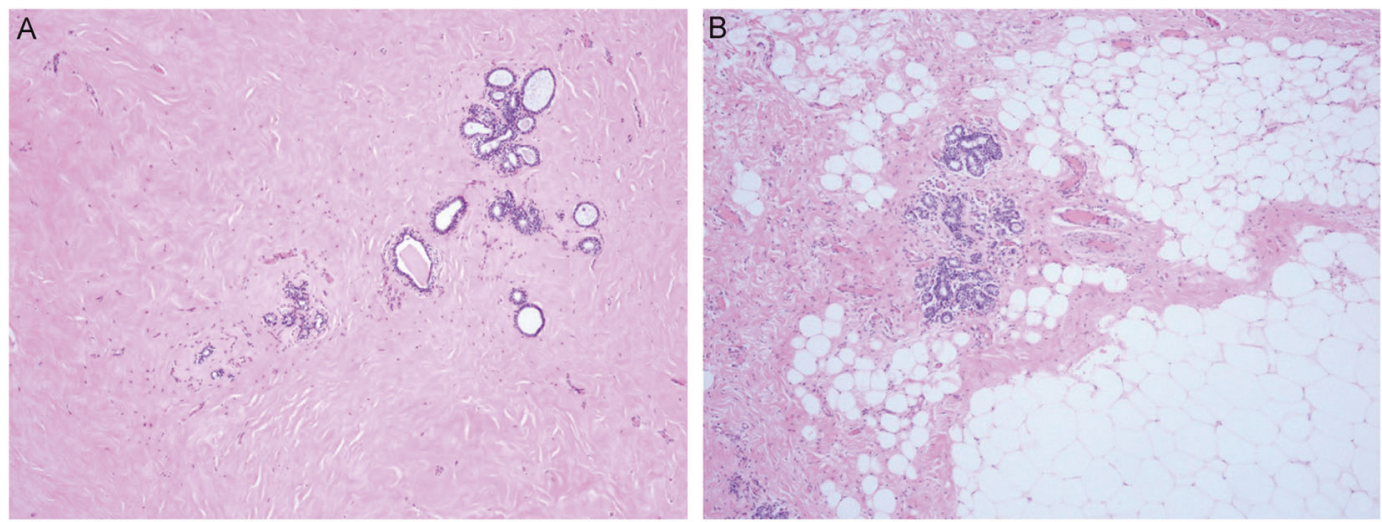

Fig. 3 Variations in breast stroma in patients with gender dysphoria. a Predominantly fibrous stroma. b Mixed stromal pattern with areas of fibrous and fatty tissue 

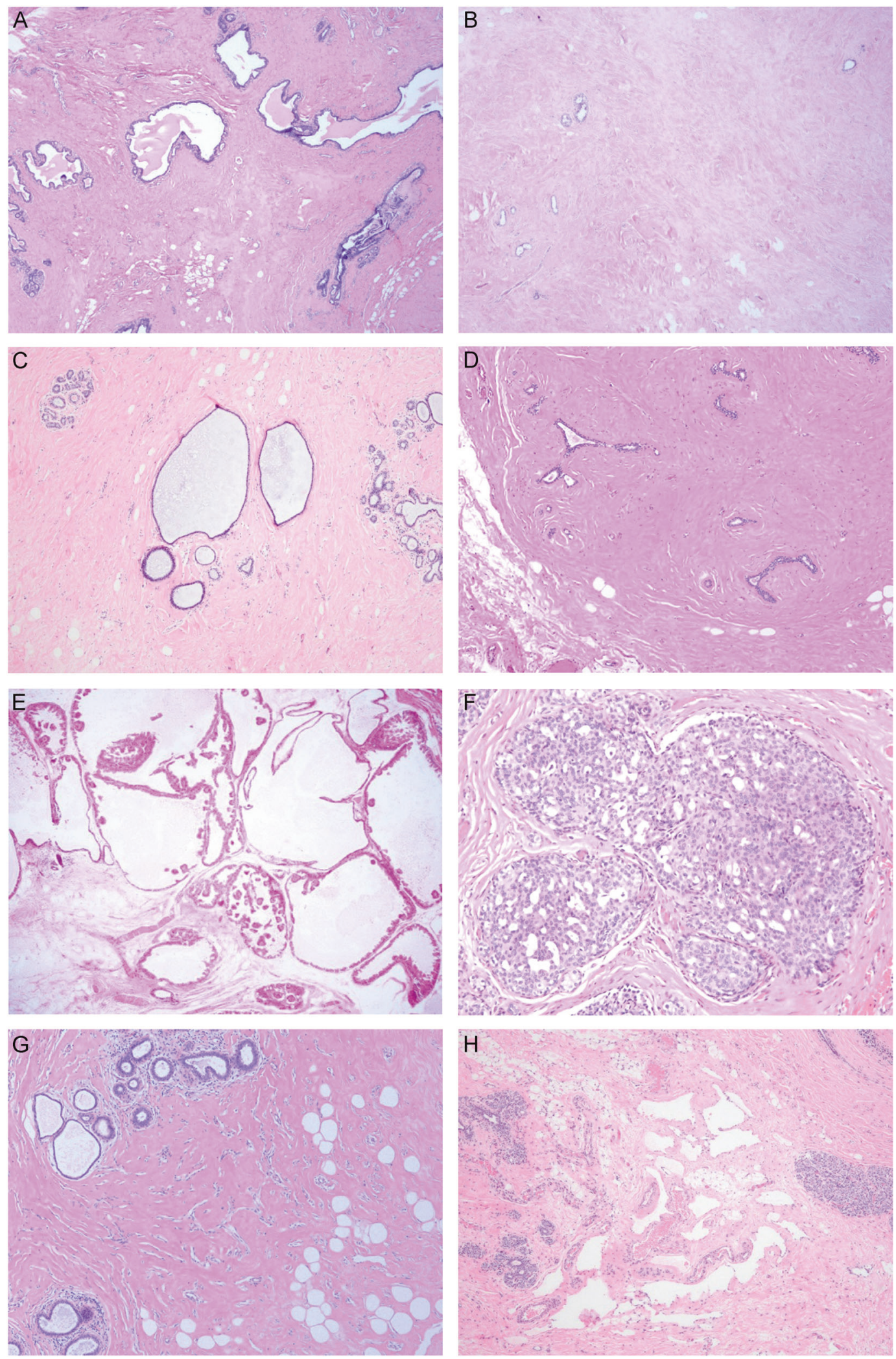

Fig. 4 Spectrum of histologic alterations in breast tissue from patients

Apocrine cysts. f Usual ductal hyperplasia. g Pseudoangiomatous with gender dysphoria. a Ectatic ducts. b Areas resembling fibrous stage of gynecomastia. c Cysts. d Fibroadenomatous change. e 

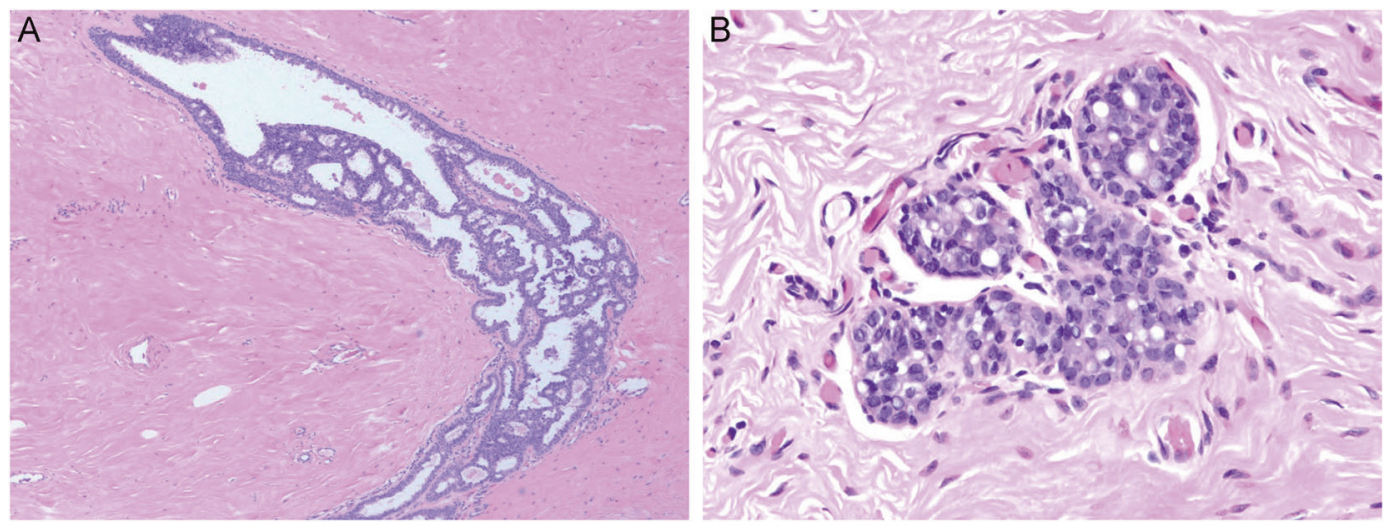

Fig. 5 Atypical lesions in breast tissue from patients with gender dysphoria. a Atypical ductal hyperplasia. b Atypical lobular hyperplasia
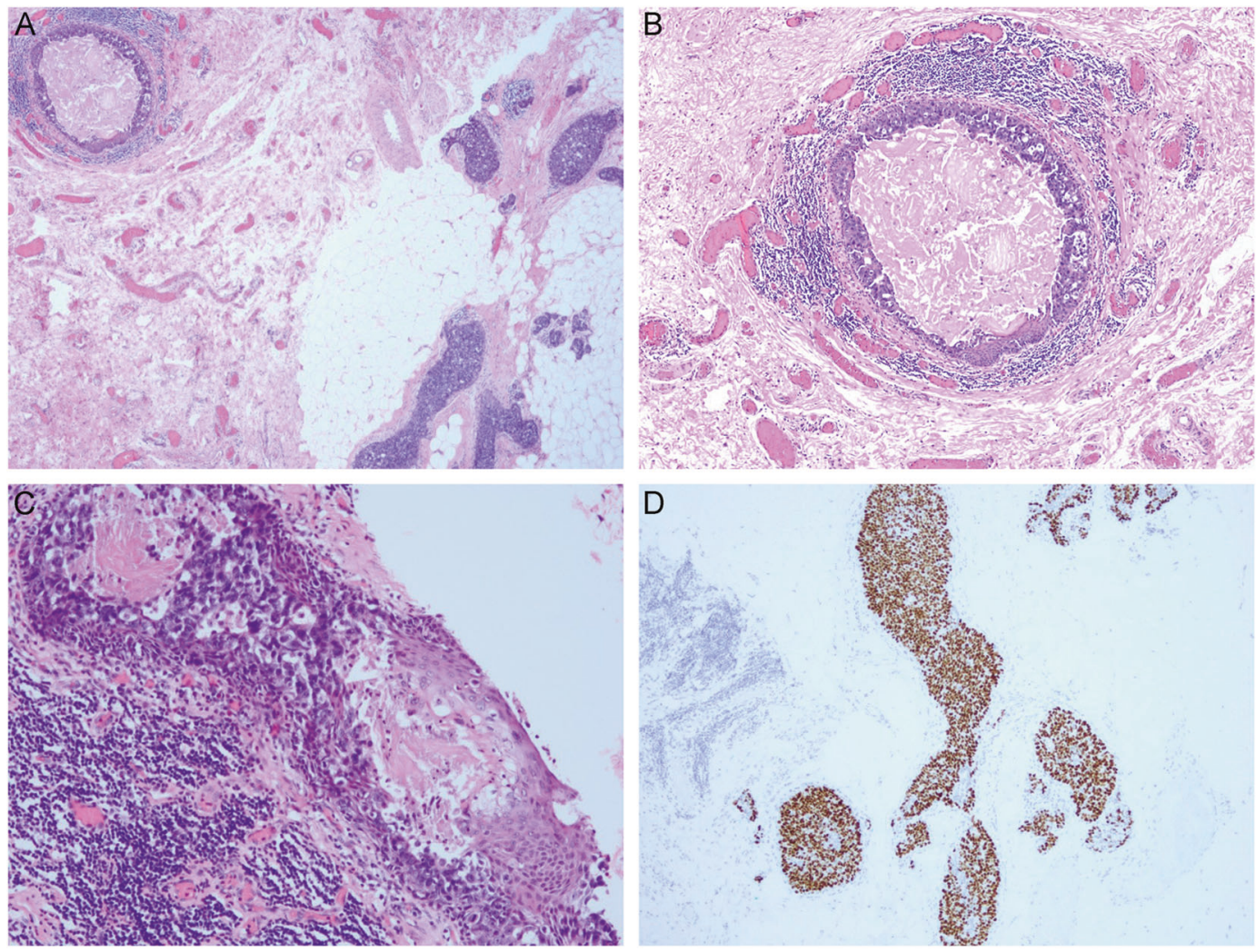

Fig. 6 Ductal carcinoma in situ, high nuclear grade, with focal squamous differentiation, from a gender dysphoric patient shown at low (a), medium (b), and high power (c). d Immunostain for estrogen receptor demonstrating strong, diffuse nuclear staining

that female-to-male transgender patients undergoing hormonal treatment will not develop breast carcinoma due to removal of their breast tissue, breast cancer has been reported in these patients, although rarely [25-30]. Thus, studying the complex physiological effects of androgen therapy as well as the proper way to handle the surgical specimens from this patient population is imperative, particularly in light of the fact that the number of individuals seeking gender reassignment surgery is increasing. Taken together, our findings and those of prior studies suggest that there is a complex interplay of androgens and estrogens, which are also generated simultaneously by the peripheral conversion of these androgens, and which may explain the prevalence and degree of lobular involution, stromal fibrosis, and gynecomastoid change seen in specimens from these patients. While we noted atypical lesions, including ductal carcinoma in situ in one patient, additional, larger studies are needed to help delineate what affect androgen therapy has on neoplasia in breast tissue. 


\section{Conclusions}

Patients with gender dysphoria are increasingly seeking gender reassignment surgeries, which typically includes mastectomy or breast reduction surgeries in female-to-male transgender patients. The histologic features seen in the breast tissue of these unique patients are the results of a complex and still poorly understood interplay of exogenous androgens administered as part of the gender reassignment process and endogenously produced hormones. Continued sampling and studying of the breast tissues of this patient population is needed to further explore these intricate physiologic processes.

\section{Compliance with ethical standards}

Conflict of interest The authors declare that they have no conflict of interest.

\section{References}

1. Flores AR, Herman JL, Gates GJ, Brown TNT. How many adults identify as transgender in the United States? Los Angeles, CA: The Williams Institute; 2016. https://williamsinstitute.law.ucla. edu/wp-content/uploads/Race-and-Ethnicity-of-Transgender-Identified-Adults-in-the-US.pdf

2. Meerwijk EL, Sevelius JM. Transgender population size in the United States: a meta-regression of population-based probability samples. Am J Public Health. 2017;107:e1-e8.

3. Canner JK, Harfouch O, Kodadek LM, et al. Temporal trends in gender-affirming surgery among transgender patients in the United States. JAMA Surg. 2018;153:609-616.

4. Frederick MJ, Berhanu AE, Bartlett R. Chest surgery in female to male transgender individuals. Ann Plast Surg. 2017;78: 249-53.

5. Monstrey SJ, Ceulemans P, Hoebeke P. Sex reassignment surgery in the female-to-male transsexual. Semin Plast Surg. 2011;25: 229-44.

6. Grynberg M, Franchin R, Dubost G, et al. Histology of genital tract and breast tissue after long-term testosterone administration in a female-to-male transsexual population. Reprod Biomed Online. 2010;20:553-8.

7. Slagter MH, Gooren LJ, Scorilas A, et al. Effects of long-term androgen administration on breast tissue of female-to-male transsexuals. J Histochem Cytochem. 2006;54:905-10.

8. Burgess HE, Shousha S. An immunohistochemical study of the long-term effects of androgen administration on female-tomale transsexual breast: a comparison with normal female breast and male breast showing gynaecomastia. J Pathol. 1993;170: $37-43$.

9. Sapino A, Pietribiasi F, Godano A, Bussolati G. Effect of longterm administration of androgens on breast tissues of female-tomale transsexuals. Ann NY Acad Sci. 1990;586:143-5.

10. Futterweit W, Schwartz IS. Histopathology of the breasts of 12 women receiving long-term exogenous androgen therapy. Mt Sinai J Med. 1988;55:309-12.

11. East EG, Gast KM, Kuzon WM Jr, et al. Clinicopathological findings in female-to-male gender-affirming breast surgery. Histopathology. 2017;71:859-65.
12. Milanese TR, Hartmann LC, Sellers TA, et al. Age-related lobular involution and risk of breast cancer. $\mathrm{J}$ Natl Cancer Inst. 2006;98:1600-7.

13. James RE, Lukanova A, Dossus L, et al. Postmenopausal serum sex steroids and risk of hormone receptor-positive and -negative breast cancer: a nested case-control study. Cancer Prev Res. 2011;4:1626-35.

14. Nicolas Diaz-Chico B, German Rodriguez F, Gonazalez A, et al. Androgens and androgen receptors in breast cancer. J Steroid Biochem Mol Biol. 2007;105:1-15.

15. Adly L, Hill D, Sherman ME, et al. Serum concentrations of estrogens, sex hormone-binding globulin, and androgens and risk of breast cancer in postmenopausal women. Int $\mathrm{J}$ Cancer. 2006;119:2702-7.

16. Danforth KN, Eliassen AH, Tworoger SS, et al. The association of plasma androgen levels with breast, ovarian and endometrial cancer risk among postmenopausal women. Int $\mathrm{J}$ Cancer. 2010;126:199-207.

17. Poulin R, Simard J, Labrie C, et al. Downregulation of estrogen receptors by androgens in the ZR-75-1 human breast cancer cell line. Endocrinology. 1989;125:392-9.

18. Garcia M, Rochefort H. Androgen effects mediated by estrogen receptors in 7,12-dimethylbenz(a)anthracene-induced rat mammary tumours. Cancer Res. 1978;33:922-3939.

19. Ip MM, Milholland RJ, Kim U, Rosen F. Androgen control of cytosol progesterone receptor levels in the MT-W9B transplantable mammary tumour in the rat. $\mathrm{J}$ Null Cancer Inst. 1982;69:673-81.

20. Zava DT, McGuire WL. Human breast cancer: androgen action mediated by estrogen receptor. Science. 1977;99:787-8.

21. Secreto G, Fariselli G, Bandieramonte G, et al. Androgen excretion in women with a family history of breast cancer or with epithelial hyperplasia or cancer of the breast. Eur J Cancer Clin Oncol. 1983;19:5-10.

22. Key T, Appleby P, Barnes I, Reeves G. Endogenous sex hormones and breast cancer in postmenopausal women: reanalysis of nine prospective studies. J Natl Cancer Inst. 2002;94:606-16.

23. Labrie F, Luu-The V, Labrie $C$, et al. Endocrine and intracrine sources of androgens in women: inhibition of breast cancer and other roles of androgens and their precursor dehydroepiandrosterone. Endocr Rev. 2003;24:152-82.

24. Missmer SA, Eliassen AH, Barbieri RL, Hankinson SE. Endogenous estrogen, androgen, and progesterone concentrations and breast cancer risk among postmenopausal women. J Natl Cancer Inst. 2004;96:1856-65.

25. Nikolic DV, Djordjevic ML, Granic M, et al. Importance of revealing a rare case of breast cancer in a female to male transsexual after bilateral mastectomy. World J Surg Oncol. 2012;10:280.

26. Burcombe RJ, Makris A, Pittam M, Finer N. Breast cancer after bilateral subcutaneous mastectomy in a female-to-male transsexual. Breast. 2003;12:290-3.

27. Shao T, Grossbard ML, Klein P. Breast cancer in female-tomale transsexuals: two cases with a review of physiology and management. Clin Breast Cancer. 2011;11:417-9.

28. Mueller A, Gooren LJ. Hormone-related tumors in transsexuals receiving treatment with cross-sex hormones. Eur J Endocrinol. 2008;159:197-202.

29. Katayama $\mathrm{Y}$, Motoki $\mathrm{T}$, Watanabe $\mathrm{S}$, et al. A very rare case of breast cancer in a female-to-male transsexual. Breast Cancer. 2016;23:939-44.

30. Gooren L, Bowers M, Lips P, Konings IR. Five new cases of breast cancer in transsexual persons. Andrologia. 2015;47: $1202-5$. 